\title{
Adenoid Cystic Carcinoma of Tongue: A Case Report \& Review of Literature
}

\author{
Hasna K. K ${ }^{1}$ Sudha.$S^{2}$, Anjana C.M ${ }^{3}$, Ismayil. $P^{4}$, Faseela Beegum P.K ${ }^{5}$
}

\begin{abstract}
Adenoid cystic carcinoma is a malignant neoplasm arising from the salivary glands. The most characteristic features of this tumor are its local aggressiveness, perineural invasion, tendency to metastasize and high recurrence rates. Here we discuss a case of Adenoid Cystic Carcinoma in a 60-year-old female presented with a swelling and ulceration on the anterolateral surface of the tongue with its clinical features, histopathology, diagnosis and treatment along with a brief review of the relevant literature
\end{abstract}

\section{Introduction}

Adenoid cystic carcinoma (AdCC) is a less common high grade malignant neoplasm that represents approximately $1-2 \%$ of all malignant neoplasms of the head and neck and $10-15 \%$ of all malignant salivary gland neoplasms ${ }^{1,2}$.Not only the salivary glands AdCC can also involve lacrimal and ceruminous glands as well as other sites in the head and neck ${ }^{3,4}$.

It occurs more often in the minor salivary glands (43-80\%) than in the major glands (20-49\%), and is found mainly in the oral cavity, followed by Sino nasal tract and oropharynx ${ }^{5}$. The most frequent intraoral subsite is the palate, followed by the buccal mucosa and the floor of the mouth. In the oropharynx, the base of the tongue and the soft palate are the most frequently involved sites ${ }^{5}$. One of the least frequent sites of presentation is the mobile part of the tongue, the incidence of only approximately $3 \%$ having being reported ${ }^{1}$. It is more common in females. The natural history of AdCC characterized by slow but relentless growth, multiple local recurrence, and distant hematogenous dissemination mainly to lung, liver bone and brain ${ }^{6}$. Lymph node metastasis is un usual. AdCC also has a high propensity for perineural invasion, sometime associated with remotely occurring lesions along nerve sheath, which significantly increase the risk recurrence after resection, even when negative surgical margin is obtained.

Salivary gland neoplasms constitute a diverse group of tumors, exhibiting different histological characteristics and variable clinical behavior pattern ${ }^{7}$. Although almost 40 histological types of SGTs exist, some are exceedingly rare ${ }^{8}$.Histopathologically, AdCC is identified as a tumor with biphasic differentiation of epithelial and myoepithelial cell (MEC), which often shows mixed patterns of cribriform, tubular and solid types ${ }^{9}$. Here we report a case of AdCC in 60yr old female patient on the anterolateral surface of mobile part of the tongue.

\section{Case Report}

A 60- year old female reported in our institution with a chief complaint of pain and ulceration on the tongue for the last one week. She had noticed an asymptomatic swelling on the same site for the last 6 months and no specific treatment was not sought for the same. Intra oral examination revealed a swelling of size $2 \mathrm{cmx}$ $1.5 \mathrm{~cm}$ on the left lateral surface of tongue which extends towards the tip of the tongue (fig 1). An ulceration of size $.5 \mathrm{~cm} x .5 \mathrm{~cm}$ was present over the swelling. The swelling was firm, mobile and tender on palpation. The overall clinical picture of the lesion was quite indolent. No significant cervical lymphadenopathy was noted and no history of paresthesia was given.

Fine needle aspiration cytology was performed. FNA yielded thick blood tinged mucinous material, and smear on histopathological examination showed acute and chronic inflammatory cells in a mucinous back ground. Since the FNAC report was inconclusive an ultrasound scan was advised. It showed a hypoechoic lesion of size $1 \mathrm{~cm} \mathrm{x} .9 \mathrm{~cm}$ on the lateral and dorsal part of the tongue. Few blood vessels were noted through the lesion, there was no calcification and no significant cervical lymphadenopathy. Then differential diagnosis of minor salivary gland neoplasm was made. The possibility of benign connective tissue neoplasm was also considered. 


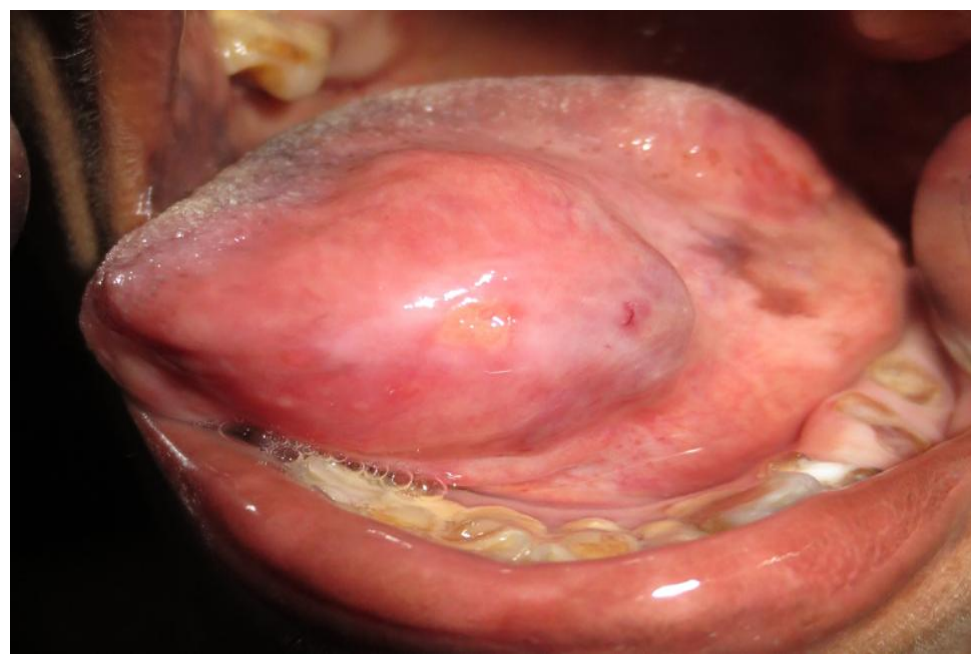

Fig 1 Intraoral view

Routine blood examination was normal and incisional biopsy was performed. Upon histopathological examination, the tissue showed tumor cell proliferation in tubular and cribriform pattern. At areas, solid nests of uniformly sized darkly staining basaloid cells were seen. In most of the areas tumor cells are punctuated by round to ovoid cyst like spaces in swiss cheese configuration (fig $2 \& 3$ ). At one area tissue showed tumor cell proliferating adjacent to the nerve bundle indicating perineural invasion (fig 5). On the basis of histopathological findings, diagnosis of adenoid cystic carcinoma was made.

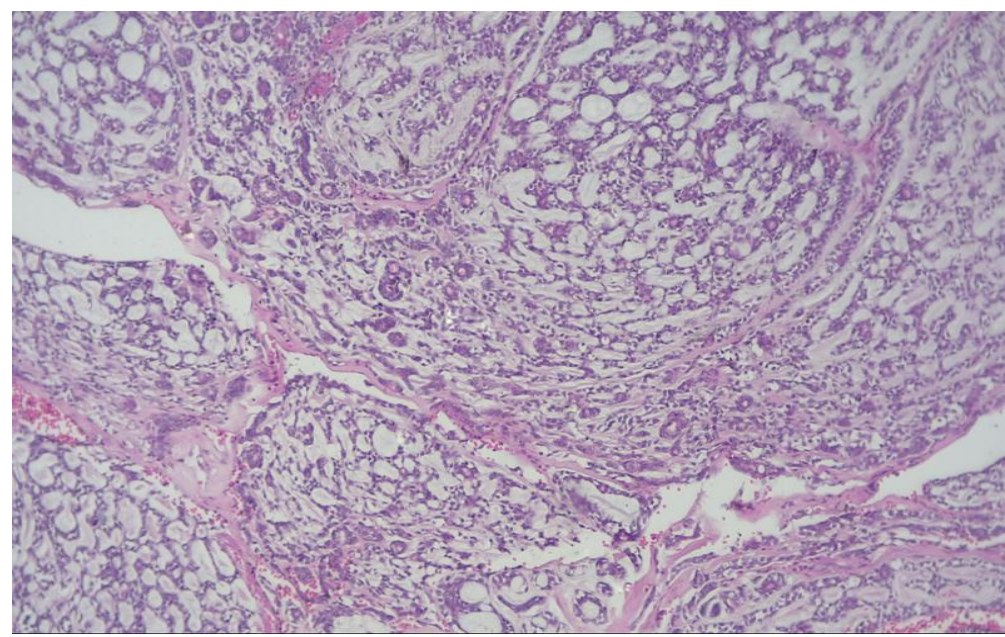

Fig 2 H \& E stained section 10 x (tumor cell proliferation in tubular and cribriform pattern)

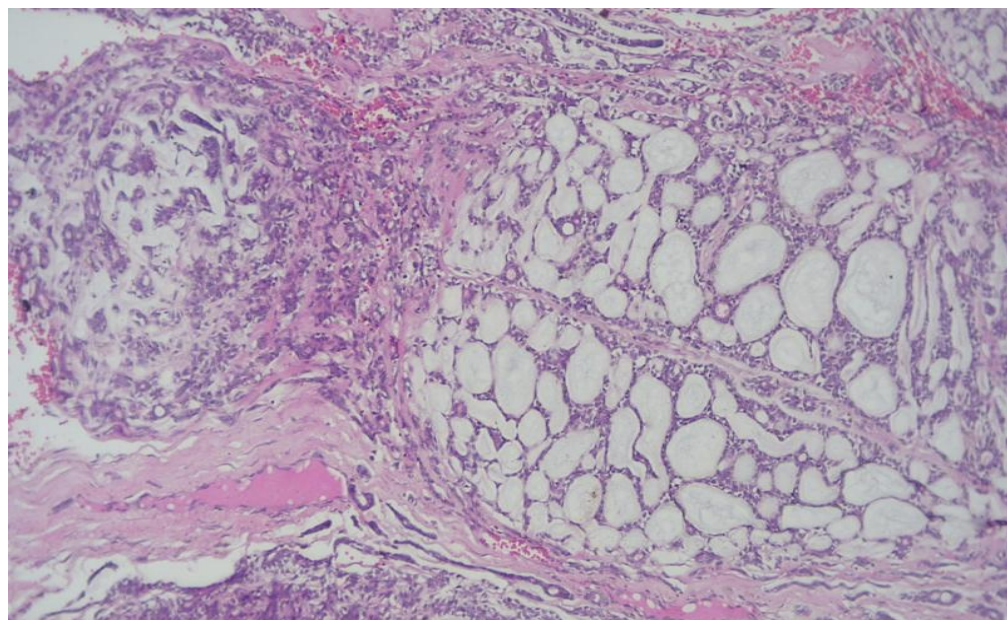

Fig 3 H\& E stained section 40x (tumor cell proliferation mainly in cribriform pattern) 


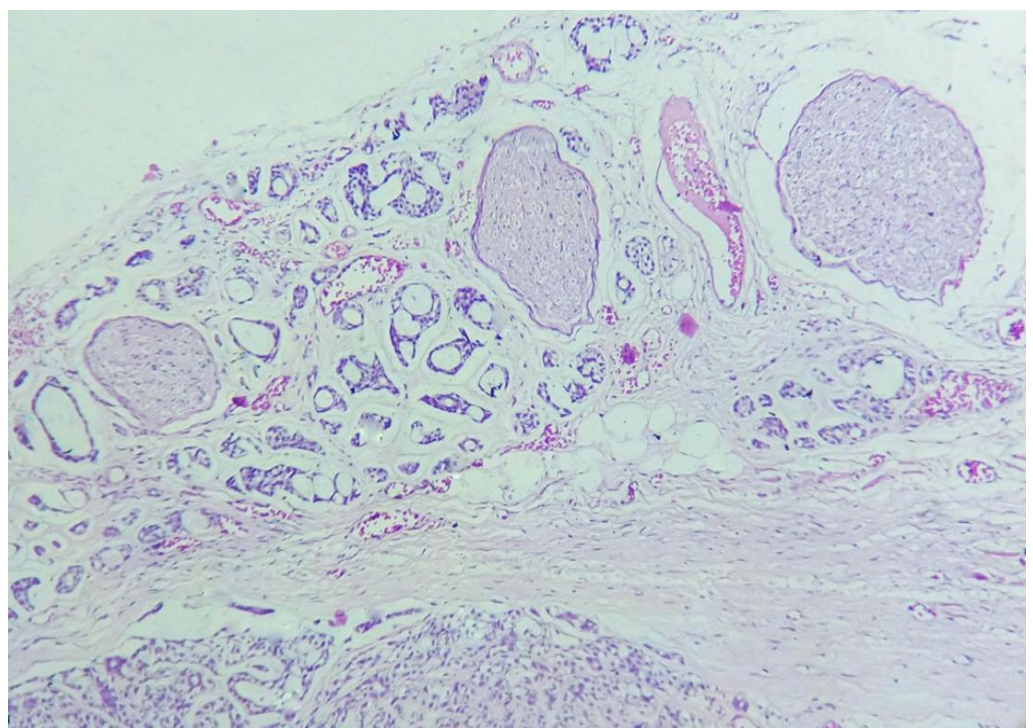

Fig 4 ( tumor cells proliferating adjacent to the nerve bundle indicating perineural invasion)

Although hematoxylin-eosin staining is still the gold standard method used for diagnosis, immunohistochemistry (IHC) can enhance the accuracy of the diagnosis. So, in order to confirm the histopathological diagnosis IHC was performed using CD 117. It yielded a strong positivity (fig 5). Then the confirmatory diagnosis of adenoid cystic carcinoma was made

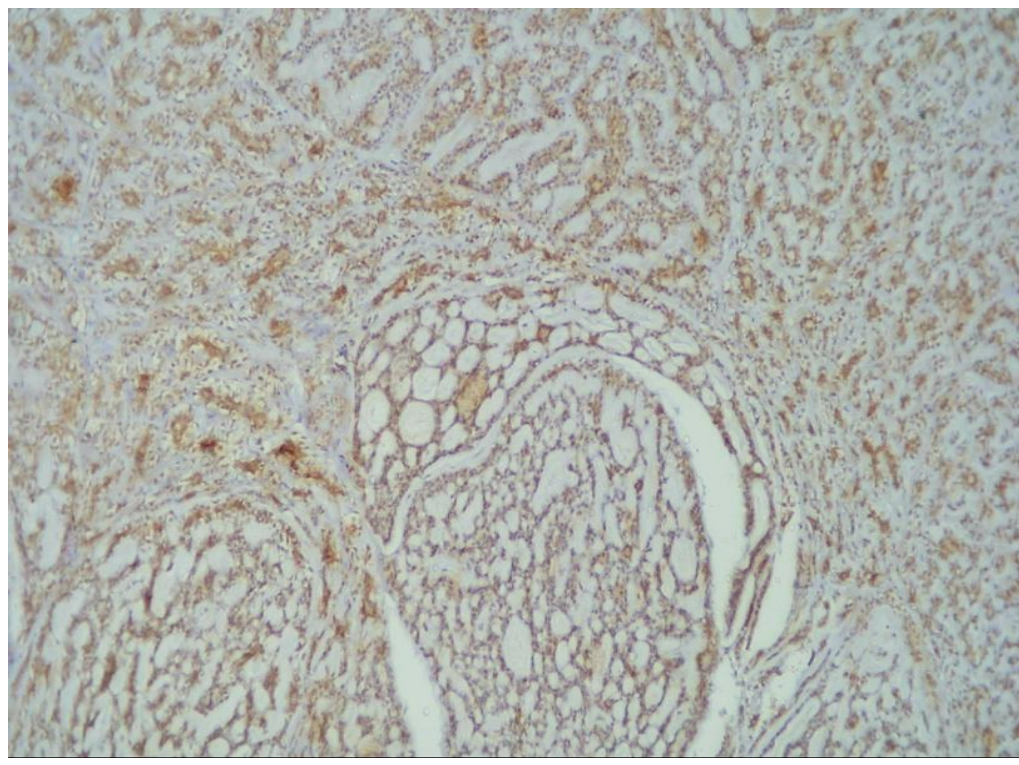

Fig 5 IHC stained section (10x) shows strong positivity for CD117

Patient was referred to the regional cancer center. Later excision of the lesion along with radical neck dissection was performed and the margins were found to be free of tumor cells. Post-operative necessary clinical and radiological investigation was performed which were within the normal limits. The patient is being followed up till date and is still free of disease

\section{Discussion}

AdCC was first described by Robin, Lorain and Laboulbene (1853 and 1854) occurring on one parotid and two nasal tumors. These authors described the characteristic cribriform arrangement of tumor cells on microscopy and noted the invasion of surrounding structures and the spread along nerves ${ }^{10,11}$. Despite the initial observations, the tumor was regarded as a variant of the benign mixed tumor. In 1856, Bill Roth suggested the term Cylindroma for this tumor. He described it as long amorphous compartments called cylinders. The current name of adenoid cystic carcinoma was introduced by Spies in 1930. The malignant nature of this tumor was finally established byDockerty and Mayo $\mathbf{1 0 , 1 2 .}$ 
The long natural history of this tumor, its affinity for perineural invasion, and its tendency for local recurrence and potential to produce distant metastasis are well known. Although it presents a widespread age distribution, peak incidence occurs predominantly among women, between the 5th and 6th decades of life. It is a slowly growing but highly invasive cancer with high recurrence rate. Lymphatic spread to local lymph nodes is rare. Hematogenous spread, however, occurs often in the course of the disease. Similar to the literature, the case that we are reporting is also seen in a female on the antero lateral side of tongue and incidence is in the 6th decade of life. But the site being the rarest.

AdCCs often exhibit mixed patterns of cribriform, tubular and solid types, classified according to the predominant pattern., the solid subtype showed more aggressive behavior and relatively poorer short-term survival when compared with other two types and considered as a high-grade lesion. In 1958, Patey and Thackray proposed that a solid growth pattern reveals a poor prognosis. Subsequently, grading of this tumor has evolved into stratification into 3 grades with increasing aggressiveness based on predominant growth pattern: tubular as Grade I, cribriform as Grade II, and solid type as Grade III. Regarding the histopathological grading of ACC, several systems have been proposed".

Szanto et al. have defined a three-tier grading system which takes into account the histomorphologic pattern and percentage of the solid component. This has been regarded as a useful prognostic indicator. Grade 1 AdCCs are well differentiated and composed of tubular and cribriform patterns without solid components; grade 2 AdCCs are characterized by a pure cribriform pattern or mixed with more than $30 \%$ of solid areas; and grade 3 AdCCs are tumors with marked predominance of the solid pattern ${ }^{13}$. Spiro recommended only when the solid parts account for more than $50 \%$ of the tumor it is considered high grade. Recently, Van Weert et al. proposed the presence of a solid pattern regardless of its quantity is a poor prognosticator. Anyway, the solid growth pattern is related to more aggressiveness, poorer short-term survival and probably higher risk of nodal metastasis and early distant metastasis ${ }^{9}$.

In many of the studies, a solid growth pattern is associated with worse prognosis, advanced stage and development of distant metastases ${ }^{10,14}$ Neural invasion can be seen even in early-stage tumors and has been regarded as an unfavorable prognostic factor, associated with distant metastasis and adverse final outcome ${ }^{10}$. Neural invasion did not predict hematogenous spread; distant metastases were related to age, primary site and nodal $(\mathrm{N})$ classification. Teymoortash et al reviewed 22 cases of AdCC with documented perineural invasion and proposed a new classification scheme for AdCC based on the presence of characteristic features in perineural invasion ${ }^{15}$. They classified tumors as $\mathrm{p} 1$ when true perineural or endo neural invasion was observed and $\mathrm{p} 2$ when tumor was adjacent to nerves without invading them. Patients with $\mathrm{p} 1$ tumors had a higher recurrence rate in comparison with $\mathrm{p} 2$ patients $^{2}$.

ACC was consistently positive for cytokeratin (CK) AE1/3, CK 34bE12, CK5/6, CK7, CK14, CK18, p63, CA19-9, c-KIT (CD117), PDGFRA, MUC1, and Ki-67, EMA, CEA, CAM5.2, p53, CD10, S-100 protein and synaptophysin and consistently negative for CK8, CK20, desmin, CD34, chromogranin, MUC2, MUC5AC and MUC6 $^{8}$. ACCs displayed well organized basal-luminal differentiation, highlighted by CK5/CK7 immunostaining. C-kit (CD 117) is a trans-membrane tyrosine kinase receptor detected in some malignant tumors but its expression in salivary gland tumors (SGTs) is rather controversial, although affinity for adenoid cystic carcinoma has been reported. Strong expression of C-kit among MSGTs was exclusive in $\mathrm{AdCC}^{\mathbf{1 6}}$. Histopathologically PLGA has close resemblance with AdCC. C Kit expression was virtually lacking in PLGA. S-100 showed higher expression in PLGA than in ACC. Also, vimentin expressed in ACC helps in distinguishing it from PLGA. According to a recent study c-kit expression in ACC highest in solid variant, followed by cribriform and tubular variant.

Recent guidelines in the management of AdCC is recommend as complete surgical resection, extended if possible, with ipsilateral lymph node dissection in case of lymphadenopathy on clinical examination or CT. Lymph node dissection is also standard in high-grade solid tumor and optional in low-grade tumor. Postoperative cervical lymph node area radiation may be indicated by pathologic analysis of the dissection specimen. Following complete resection, radiation therapy is indicated both in high-grade and low-grade tumour.Adenoid cystic carcinomas have a variable prognosis. The 5 -year survival rate is $75 \%$, but 10 -year survival rate is only $20 \%$, and survival at 15 years is about $10 \%$.

\section{Conclusion}

Early diagnosis and proper treatment of this less common neoplasm of the tongue are important from a functional point of view, since AdCC are slowly growing and may produce diffuse invasion into adjacent structures . Surgery is still the cornerstone of treatment and radiotherapy is indicated in the presence of compromised surgical margins. Long-term follow-up is essential. 


\section{References}

[1]. Kumar S, Agarwal P, Nimmi V. ScienceDirect Adenoid cystic carcinoma: A rare late presentation of the mobile tongue. J Oral Biol Craniofacial Res. Craniofacial Research Foundation; 2016;4-7.

[2]. Kim KH, Sung MW, Chung PS, et al. Adenoid cystic carcinoma of the head and neck. Arch Otolaryngol Head Neck Surg (US). 1994;120:721-726.

[3]. Ross W. Green, MD, Uchechukwa C. Megwalu, MD Adenoid cystic carcinoma of the external ear: a population based study, American journal of otolaryngology- head and neck medicine and surgery 37(2016) 346-350

[4]. Hellquist HB, Skalova A. Histopathology of the Salivary Glands. Adenoid Cystic Carcinoma. Springer; $2014221-60$.

[5]. Barnes L, Silver CE, Rodrigo JP, Shah JP, Triantafyllou A, Rinaldo A, et al. Cervical lymph node metastasis in adenoid cystic carcinoma of oral cavity and oropharynx: A collective international review §. 2016

[6]. Umeda M, Komatsubara H, Nishimatsu N, et al. Establishment and characterization of human adenoid cystic carcinoma line of the salivary gland which is serially transplantable and spontaneously metastasises to the lung in nude mice. Oral Oncol (US). 2002;38: $30-34$.

[7]. Garg V, Roy S, Surya K, Preeti K, Bakshi S, Chauhan I. Adenoid Cystic Carcinoma of Buccal Mucosa: A Rare Case Report. Indian J Otolaryngol Head Neck Surg [Internet]. Springer India; 2015;1-4

[8]. Namboodiripad PCA. A review: Immunological markers for malignant salivary gland tumors. J Oral Biol Craniofacial Res [Internet]. Elsevier Ltd; 2014;4(2):127-34.

[9]. Du F, Zhou C, Gao Y. Annals of Diagnostic Pathology Myoepithelial differentiation in cribriform, tubular and solid pattern of adenoid cystic carcinoma: A potential involvement in histological grading and prognosis. Ann Diagn Pathol [Internet]. Elsevier Inc.; 2016; 22:12-7

[10]. Coca-pelaz A, Rodrigo JP, Bradley PJ, Vander V, Rinaldo A, Haigentz M, et al. Adenoid cystic carcinoma of the head and neck An update q. 2016;51(2015):652-61 2057-67.

[11]. Stell PM. Adenoid cystic carcinoma. Clin Otolaryngol Allied Sci 1986;11: 267-91.

[12]. Dockerty MB, Mayo CW. Primary tumors of submaxillary gland with special reference to mixed tumors. Surg Gynecol Obstet 1942; 74:1033-45.

[13]. Szanto PA, Luna MA, Tortoledo ME, White RA. Histologic grading of adenoid cystic carcinoma of the salivary glands. Cancer 1984; 54:1062-9.

[14]. Vander Poorten VLM, Balm AJM, Hilgers FJM, et al. The development of a prognostic score for patients with parotid carcinoma. Cancer 1999;85: 2057-67.

[15]. Teymoortash A, Zieger L, Hoch S, Pagenstecher A, Hofer MJ. Distinct microscopic features of perineural invasion in adenoid cystic carcinoma ofthe head and neck. Histopathology 2014; 64:1037-9.

[16]. Bamidele Kolude, Akinyele Olumuyiwa Adisa, Ahmed Oluwatoyin Lawal*, Bukola Folasade Adeyemi, Strong immunohistochemical expression of C-kit may characterize adenoid cystic carcinoma of the salivary gland: Journal of Oral and Maxillofacial Surgery, Medicine, and Pathology 26 (2014) 549-553

[17]. Gondivkar SM, Gadbail AR, Chole R, Parikh RV (2011) Adenoid cystic carcinoma: a rare clinical entity and literature review. Oral Oncol 47:31-36 